\title{
Recent Epidemiological Profile of Cutaneous Leishmaniasis in Iranian Military Personnel
}

\author{
Elnaz Tabibian ${ }^{1}$, Seyyed Javad Hosseini Shokouh ${ }^{2}$, Shahram Rahimi Dehgolan ${ }^{1}$, Arasb \\ Dabbagh Moghaddam ${ }^{3}$, Mohsen Tootoonchian ${ }^{3}$, Mahtab Noorifard ${ }^{2, *}$ \\ ${ }^{1}$ Tehran University of Medical Sciences, Tehran, IR Iran \\ 2 Infectious Diseases Research Center, Aja University of Medical Sciences, Tehran, IR Iran \\ ${ }^{3}$ Preventive Medicine Department, Deputy of Health, Aja University of Medical Sciences, Tehran, IR Iran \\ ${ }^{*}$ Corresponding author: Mahtab Noorifard, Aja University of Medical Sciences, Etemad St., Fatemi St., Tehran, IR Iran. Tel: +98-2185952993, Fax: +98-2185952993, E-mail: mahtab_noori- \\ fard@yahoo.com.
}

Received: October 21, 2013; Revised: December 10, 2013; Accepted: December 14, 2013

\begin{abstract}
Background: Leishmaniasis is one of the zoonotic diseases caused by protozoa of trypanosomatidae family. The cutaneous type with various manifestations is endemic in Iran and affects people of many provinces. Outbreaks may occur in military personnel who deploy these endemic areas.

Objectives: The main objective of this study was to evaluate the most important epidemiologic features of leishmaniasis in Iranian military personnel from 2005-2010.

Patients and Methods: This was a cross-sectional study. Data was gathered from the regional passive surveillance system of the Iranian Army and double checked by the Deputy of Health of Aja University of Medical Sciences. Analysis was performed by the STATA Software using appropriate statistical functions.

Results: During the 6 years of study, 3767 cases of cutaneous leishmaniasis were reported, 15\% of the total reported infectious diseases (excluding upper respiratory infections, which were not included in this study). Cutaneous leishmaniasis was the third most prevalent infectious disease. Sixty nine percent of cases were reported in autumn. Most were related to three provinces: Isfahan, Khuzestan and Bushehr. Two thirds of the reports were from the Air Force.

Conclusions: There has been a decline in the overall trend of cutaneous leishmaniasis in the recent years, but there are still peaks in some provinces particularly during autumn. Army health commanders must do their best to prevent the disease and control its cycle, especially in high endemic areas.
\end{abstract}

Keywords:Leishmaniasis, Cutaneous; Military Personnel; Epidemiology; Iran

\section{Background}

Cutaneous leishmaniasis (CL, known as "Salak" (means: a year) among the Iranian population) is one of the top 10 diseases of the tropical world. It is also an endemic disease in Iran. This zoonotic disease has 3 forms: cutaneous, visceral (Kala-azar), and mucocutaneous. Insect (sand fly Phelebotomus) bite is the main source of disease transmission. Purplish ulcer with central necrosis in exposed areas such as face and extremities which can last up to 1-2 years, is a pathognomonic sign for $\operatorname{CL}(1,2)$.

Twenty million people are infected worldwide, and its incidence is 400,000 yearly. For definite diagnosis, clinicians should visualize the amastigote form of the protozoa in specimens, but PCR and lymphatic system biopsy (including bone marrow, lymph node, etc.) could also be useful. The visceral form (Kala-azar) is epidemic in India and Sudan and some parts of Iran ie: Meshkin Shahr and Fars Province, but the cutaneous type has a high preva- lence worldwide (3-5). About 90\% of CL cases have been reported from seven countries, including, Saudi Arabia, Afghanistan, Algeria, Iran, Brazil, Peru, and Iraq. Disability adjusted life years (DALY) of leishmaniasis is about 2 million years (6-9).

In military centers due to the presence of predisposing and precipitating factors, pattern of cutaneous diseases is quite different from the community. These important factors include, lesser number of baths and poor hygiene among young soldiers particularly those with lower level of literacy, contact with soil and dirty water during military maneuvers and so on (10).

In USA military force burden of cutaneous diseases is about 3 million people/d which is a shocking number in any military system (11). Among these cutaneous diseases, CL is specifically important, because it has very special endemic areas. Although CL may be widespread on a 
global scale, it is almost always localized because of the specific habitat requirements of the sand fly vector and its various reservoir hosts (12). So outbreaks may occur in military personnel and other travelers from non-endemic countries who have visited endemic areas and have become exposed. For example in Afghanistan and Iraq non-habitant soldiers and military personnel of western countries are at excessive risk of CL. This issue can complicate epidemiological features of the disease and should be considered for all military service and migration affairs even between two provinces of a country (13-15).

According to recent reports from the Iranian Health Ministry, CL with an incidence rate of 20,000 cases per year has the second place among parasitic diseases (malaria is still the first). During the last decade incidence of CL has been increasing; from 2000 to 2004 we had a $105 \%$ increase in the number of CL patients $(16,17)$. Thus, currently CL has again become an important health issue in Iran and has not yet been completely controlled. Although today we know much more than a decade ago, but we are not closer to the prevention or control of this neglected disease (18).

\section{Objectives}

Considering that there has not been any comprehensive study about the epidemiologic features of leishmaniasis in the Iranian Army, this study was designed to evaluate the epidemiologic profile of CL in Iranian military personnel.

\section{Patients and Methods}

The main objective of this cross-sectional study was to assess the epidemiologic features of $\mathrm{CL}$ in the Iranian Army from 2005 to 2010 . We should emphasize that reporting of leishmaniasis is mandatory in the surveillance system of the Iranian Army. So at the end of each month we were provided with the reports of CL cases in all provinces and in triple Army forces. Therefore based on the population of each center as the denominator we were able to calculate the cumulative incidence rate for those 3 months.

Data was collected from archived leishmaniasis records in preventive medicine section of Deputy of Health of Aja University of Medical Sciences in Tehran. Our inclusion criterion was any military person with confirmed diagnosis of CL (in this study family members of army personnel were not included). Diagnosis of all cases had been confirmed by a physician at each center. In controversial cases ulcer biopsy and amastigote visualization of specimen was the confirmatory paraclinic measure. Data analysis was based on t-test and ANOVA for quantitative and chi2 for qualitative variables using the 11th version of the STATA software.

\section{Results}

In 62,048 reported Infectious cases from 25 provinces in 2005-2010, 3767 (15\%) were related to CL (of course upper respiratory infections were not included in this study). CL was the third most prevalent reported disease (fungal disease and scabies were in the first and second places), while visceral leishmaniasis (VL) was a rare infection in our country (only 2 cases of VL were reported during the 6 years).

Distribution of CL in the Iranian Army for these 6 years is illustrated in Figure 1. With the assumption that the denominator of incidence rate (total population) would not have major changes, we can say that $C L$ incidence rate has been decreased from 2005 to 2010. Most cases were reported from Isfahan, Khuzestan, Bushehr followed by Zanjan, Ghazvin and Khorasan Razavi provinces (Figure 2). The geographical distribution of CL in different provinces has been illustrated in Figure 3. Distribution of CL in triple forces is illustrated by Figure 4 . The most prevalent disease in the Air Force was CL, and this difference was significant $(\mathrm{P}=0.001)$.

Seasonal distribution of CL is illustrated in Figure 5. During all seasons, scabies and fungal diseases were the first and second most prevalent, but in autumn CL had a remarkable peak (Table 1), and its cumulative incidence rate had a significant association with season $(\mathrm{P}=0.001)$.

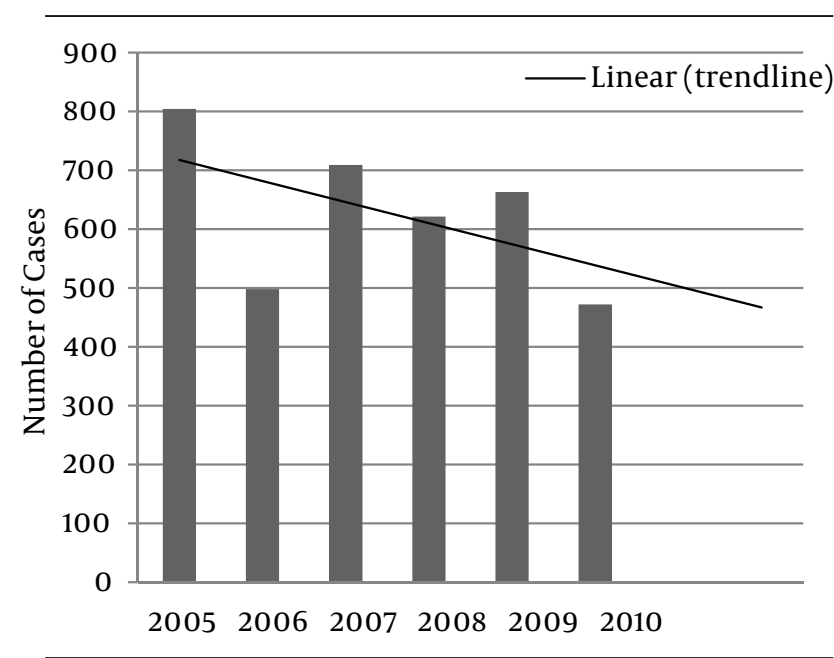

Figure 1. Annual Trend of Cutaneous Leishmaniasis in Iranian Military Personnel

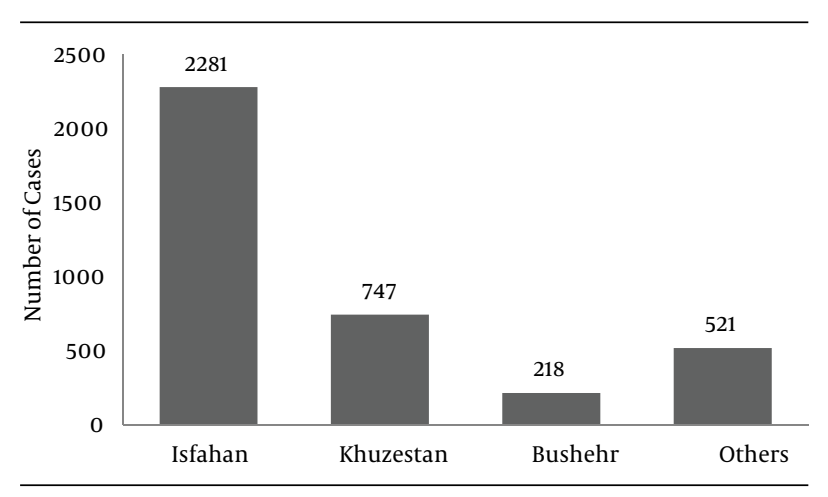

Figure 2. Distribution of Cutaneous Leishmaniasis in Different Provinces 
Tabibian E et al.

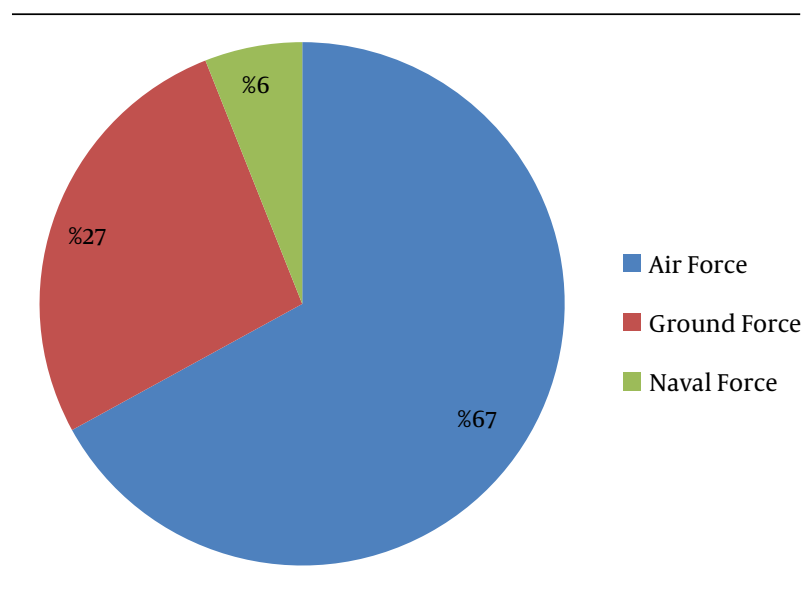

Figure 3. Geographical Distribution of Cutaneous Leishmaniasis

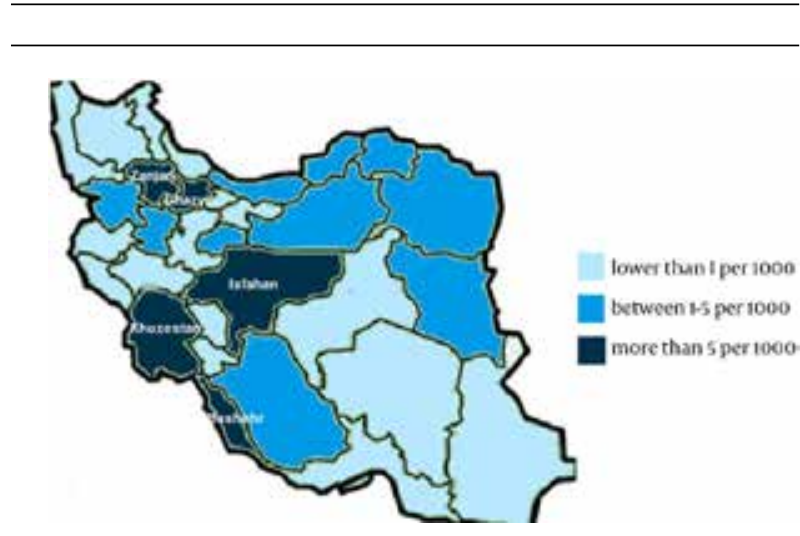

Figure 4. Distribution of Cutaneous Leishmaniasis Among Triple Army Forces

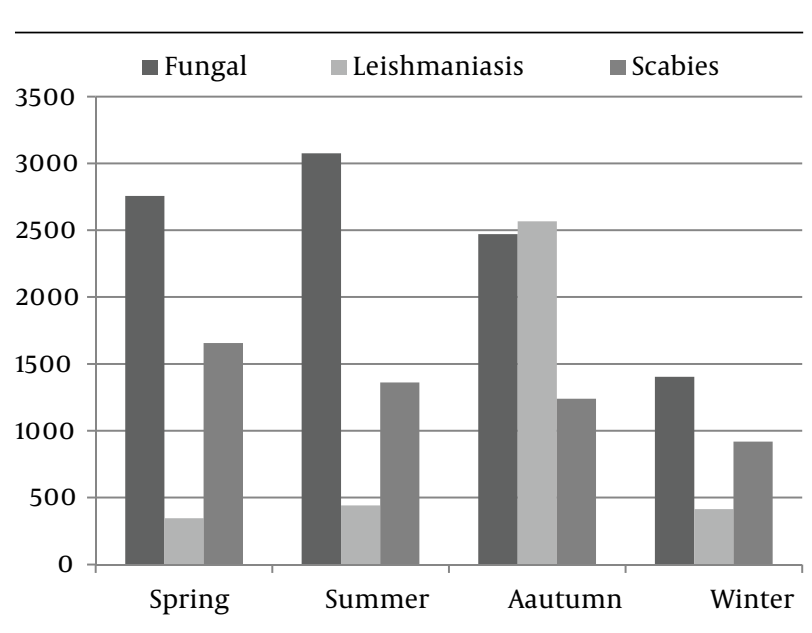

Figure 5. Distribution of the Top Three Infectious Diseases During Different Seasons

For more accuracy, P value $<0.01$ was considered as the significance level and based on this measure, differences of CL incidence rates among triple forces and seasons were strongly significant.
Table 1. Seasonal Distribution of Cotaneus Leishmaniasis

\begin{tabular}{lll}
\hline Season & No. & $\%$ \\
\hline Spring & 346 & 9 \\
Summer & 441 & 11 \\
Autumn & 2567 & 69 \\
Winter & 413 & 11 \\
\hline
\end{tabular}

\section{Discussion}

In this study cumulative incidence rate of CL was about 500 persons per year (3767 cases during 6 years) which confirms Davoodi's study results (10). Among provinces as hypothesized, Isfahan and Khuzestan provinces constitutedthe first and second most prevalences followed by Bushehr and Zanjan provinces; these findings are similar to that reported by Zahirnia et al. (19).

We also found that two thirds of the patients were members of the Air Force, although its population is only $19 \%$ of the entire Army. So the incidence rate in the Air Force is 7 times greater than the other forces of the army. We think that this difference is due to the presence of an air force base in Isfahan, which is the most prevalent province.

Sixty nine percent of our cases had occurred during autumn. This difference is explained by the disease cycle. The transmission of the protozoa from rodents to humans occurs at the end of the sand fly season (September-October) and after a short incubation period of one week to two months, the lesions appear in humans in late autumn (20).

We can conclude that since CL is a debilitating disease causing military personnel to take time off from work and since it has no FDA-approved vaccine or prophylactic medication so far (21), more preventive programs should be considered. Control measures against vectors or reservoir hosts of infection might be effective in particular settings $(22,23)$. Personal protective measures to decrease risk of infection include: avoiding areas where CL is endemic (if possible), using permethrin-treated bed nets and clothing, minimizing the amount of exposed skin or applying insect repellents to exposed areas.

These results and conclusions are needed to be announced to the army health commanders for better planning in health facilities of different provinces to reach the goal of better control of this preventable disease.

\section{Acknowledgements}

We thank all the personnel of Preventive Medicine Department of Aja University of Medical Sciences and also especially Ms. Zeinab Rasouli and Ms. Farkhonde Rafiei.

\section{Authors' Contribution}

Manuscript writing and editing, statistical analysis: Tabibian, Rahimi Dehgolan. Data gathering: Hosseini Shookouh. Data source: Dabbagh Moghaddam. Coordinator: Tootoonchian, Corresponding author: Noorifard. 


\section{Financial Disclosure}

The authors declared that there were no financial conflicts of interest.

\section{Funding/Support}

This study was supported by Aja University of Medical Sciences.

\section{References}

1. Ministry of Health and Medical Education. Instruction of leishmaniasis Control. Tehran: Center for disease control; 1999. Available from: http://www.behdasht.gov.ir/.

2. Markle WH, Makhoul K. Cutaneous leishmaniasis: recognition and treatment. Am Fam Physician. 2004;69(6):1455-60.

3. Murray HW, Berman JD, Davies CR, Saravia NG. Advances in leishmaniasis. Lancet. 2005;366(9496):1561-77.

4. Mehregan DR, Mehregan AH, Mehregan DA. Histologic diagnosis of cutaneous leishmaniasis. Clin Dermatol.1999;17(3):297-304.

5. Bailey MS, Lockwood DN. Cutaneous leishmaniasis. Clin Dermatol. 2007;25(2):203-11.

6. World Health Organization. Expert Committee on the Leishmaniases . The Leishmaniases: Report of T a WHO Expert Committee World Health Organization; 1984.

7. WHO Expert Committee on the Control of the Leishmaniases. Meeting. Control of the leishmaniases: report of a WHO expert committee. World Health Organization; 1990.

8. Ashford RW. Leishmaniasis reservoirs and their significance in control. Clin Dermatol.1996;14(5):523-32.

9. Desjeux P. Disease watch focus: leishmaniasis. Nature Rev Microbiol. 2004;2:692-3.

10. Davoudi SM, Karimi Zarchi AA, Zafariyan S. [Prevalence of cutaneous diseases and related factors in a non-educational military center]. Iran J of Mil Med. 2011;13(1):31-5.

11. Schultz JR, Bell KM, Naugle AE, Polusny MA. Child sexual abuse and adulthood sexual assault among military veteran and civilian women. Mil Med. 2006;171(8):723-8.

12. Sanchez JL, Diniega BM, Small JW, Miller RN, Andujar JM, Weina PJ, et al. Epidemiologic investigation of an outbreak of cutaneous leishmaniasis in a defined geographic focus of transmission. Am JTrop Med Hyg. 1992;47(1):47-54.

13. Bailey MS, Caddy AJ, McKinnon KA, Fogg LF, Roscoe M, Bailey JW, etal Outbreak of zoonotic cutaneous leishmaniasis with local dissemination in Balkh, Afghanistan.J R Army Med Corps. 2012;158(3):225-8.

14. Harms G, Schonian G, Feldmeier H. Leishmaniasis in Germany. Emerg Infect Dis. 2003;9(7):872-5.

15. Weina PJ, Neafie RC, Wortmann G, Polhemus M, Aronson NE. Old world leishmaniasis: an emerging infection among deployed US military and civilian workers. Clin Infect Dis. 2004;39(11):1674-80.

16. Anker M, Schaaf D. WHO report on global surveillance of epidemicprone infectious diseases. World Health Organization; 2000. Available from: http://www.who.int/csr/resources/publications/surveillance/WHO_CDS_CSR_ISR_2000_1/en/.

17. Ershadi MR, Zahraei-Ramazani AR, Akhavan AA, Jalali-Zand AR, Abdoli $\mathrm{H}$, Nadim A. Rodent control operations against zoonotic cutaneous leishmaniasis in rural Iran. Ann Saudi Med. 2005;25(4):309-12.

18. Alvar J, Yactayo S, Bern C. Leishmaniasis and poverty. Trends Parasitol. 2006;22(12):552-7.

19. Zahirnia AH, Moradi AR, Norozi NA. [Epidemiological Survey of Cutaneous Leishmaniasis in Hamadan Province 2002-2007]. Sci J Hamadan Univ Med. 2009;16(1):43-7.

20. WHO-EMRO Country activities. Neglected tropical diseases. Cutaneous leishmaniasis in Morocco. 2013. Available from: www.emro.who.int/.

21. Herwaldt BL. Leishmaniasis. Lancet. 1999;354(9185):1191-9.

22. WHO. Communicable disease profile for Iraq. 2003. Available from: http://www.who.int/infectious-disease-news/1profile.pdf.

23. Desjeux P. Leishmaniasis. Public health aspects and control. Clin Dermatol.1996;14(5):417-23. 